\title{
A generalization of Hyers-Ulam stability on $m$-semigroups
}

\author{
Adina Pop and Maria S. Pop
}




\title{
A GENERALIZATION OF HYERS-ULAM STABILITY ON $m$-SEMIGROUPS
}

\author{
ADINA POP AND MARIA S. POP
}

Received 24 September, 2013

\begin{abstract}
In this paper we investigate the generalized Hyers-Ulam stability of mappings of $m$-semigroups $m \in \mathbb{N} ; m \geq 2$ into Banach spaces. For $m=3$ the results can be found in Amyari and Moslehian [Approximate homomorphisms of ternary semigroups, Lett. Math. Phys. 77 (2006), 1-9] with the mention that they are true in the class of normal $m$-semigroups which is larger than the class of commutative $m$-semigroups. For $m=2$ we find certain results of Hyers [On the stability of the linear functional equation, Proc. Nat. Acad. Sci. USA, 27 (1941), 222224 ], Aoki [On the stability of the linear transformation in Banach spaces, J. Math. Soc. Japan, 2 (1950), 64-66], Rassias, Th. M. [On the stability of the linear mapping in Banach space, Proc. Amer. Math, Soc. 72 (1978), 297-300 ] and Rassias, J. M. [ Solution of a Problem of Ulam, J. Approx. Theory Math. 57 (1989), 268-273 ]. In addition, we establish the superstability of $m$-ary homomorphims into Banach algebras endowed with multiplicative norms, generalizing the results of Szekelyhidi [On a theorem of Baker, Lawrence and Zorzitto, Proc. Amer. Math. Soc., 84 (1982), 95-96 ] and Amyari and Moslehian (2006) .
\end{abstract}

2010 Mathematics Subject Classification: 39B52; 39B82; 46B99; 20N15

Keywords: $m$-semigroup, stability of mappings, superstability, approximately mappings, Banach algebras

\section{INTRODUCTION}

In 1940 S.M. Ulam [22] proposed the famous problem "Give conditions in order for a linear mapping near an approximately linear mapping to exist". More precisely, given a group $(G, \cdot)$, a metric group $\left(G^{\prime}, \cdot, d\right)$ and a positive number $\varepsilon>0$, does there exist $\delta>0$ such that if a function $f: G \rightarrow G^{\prime}$ satisfies the inequality $d(f(x$. $y), f(x) \cdot f(y))<\delta$ for all $x, y \in G$, then there is a homomorphism $T: G \rightarrow G^{\prime}$ such that $d(f(x), T(x))<\varepsilon$ for all $x \in G$ ? When this problem has a solution, we say that the homomorphisms from $G$ to $G^{\prime}$ are stable.

In 1941 D.H. Hyers [13] solved the Ulam problem for the case of approximate additive mappings under the assumption that $G$ and $G^{\prime}$ are Banach spaces. This result was generalized by T. Aoki [3] in 1950 and improved by Th. M. Rassias [19] in 1978. After 1982 J. M. Rassias [17, 18] , Th. M. Rassias [20] and L. Szekelyhidi [21] established the stability for Ulam problem for different mappings. The aspect 
of stability that was introduced in these papers is called Hyers-Ulam (shortly H-U) stability. During past few years several mathematicians, like P. Găvruţa [12], Z. Gajda [10] have published on various generalizations and applications of $\mathrm{H}-\mathrm{U}$ stability to a number of functional equations .

In 2006 Amyari and M. S. Moslehian [1] generalized the H-U stability of mappings on commutative ternary semigroup homomorphisms into Banach spaces. Also, the superstability ("stronger" than the concept of stability) of ternary semigroup homomorphisms into Banach algebras endowed with multiplicative norms has been investigated.

In 2012 M. Dehghanian and M. S. Modarres [6] investigated the generalized H$\mathrm{U}$ stability of ternary $\gamma$-homomorphisms on ternary semigroups. The stability of ternary algebras homomorphisms has been studied by I. S. An and C. Park [2] , M. S. Moslehian and L. Szekelyhidi [15], also.

The object of the present article is thus to formulate and solve some stability problems for $m$-ary case, in such a way to retrieve some known results from the binary or ternary case. $m$-Ary algebraic structures have many applications in different branches. For example, in the theory of automata [11], some $m$-groupoids are applied in the theory of quantum groups, ternary structures [14] and $m$-Lie algebras are used in physics. The first paper focused on $m$-groups was written by W. Dörnte [7] in 1928. In 1940 E. Post [16] published an extensive study of $m$-groups. The other mathematicians like W. Dudek [8], K. Glazek [9] investigated different $m$-ary structures.

In this paper, using a sequence of Hyers type, we generalize the H-U stability of mappings on $m$-semigroups, $m \geq 2$ into Banach spaces. As a particular case, for $m=2$ we obtain the results of P. Găvruţă [12], D. H. Hyers [13], T. Aoki [3], Th. M. Rassias [19, 20], J. M. Rassias [18], and for $m=3$ we find those of M. Amyari and M. S. Moslehian [1] with the mention that they are true in the class of normal $m$-semigroups which is larger than the class of commutative $m$-semigroups. In addition, we establish the superstability of $m$-ary homomorphims into Banach algebras endowed with multiplicative norms, generalizing the results of L. Szekelyhidi [21] and Amyari and Moslehian [1].

\section{PReliminaries}

First, we recall notations, definitions and properties related to $m$-semigroups which are used throughout this paper.

Let $\left(S,()_{\circ}\right)$ be an $m$-semigroup, $m \in \mathbb{N} ; m \geq 2$, i.e. a set $S$ with associative operation ()。: $S^{m} \rightarrow S$. A sequence of simbols $x_{1} x_{2} \ldots x_{i}$ whether they are sets or individual elements, will be abbreviated to $x_{1}^{i}$. When $x_{1}=x_{2}=\ldots=x_{i}=x$, then we will write $x_{1} x_{2} \ldots x_{i}=\stackrel{(i)}{x}$. With this convention, the associative law is written as

$$
\left(\left(x_{1}^{m}\right) \circ x_{m+1}^{2 m-1}\right)_{\circ}=\left(x_{1}^{i}\left(x_{i+1}^{i+m}\right) \circ x_{i+m+1}^{2 m-1}\right) \text { 。 }
$$


for all $x_{1}, \ldots, x_{2 m-1} \in S$ and $i=1,2, \ldots, m-1$.

Recursively, one may also define $x^{[0]}=x, x^{[1]}=(\stackrel{(m)}{x})$ o, $x^{[k+1]}=\left(x^{[k]} \stackrel{(m-1)}{x}\right)$ 。

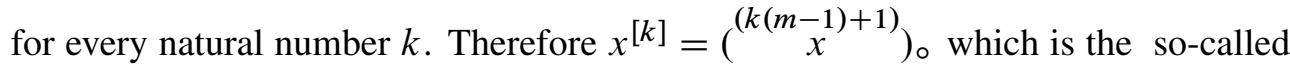
long product.

The following laws are true:

$$
\begin{gathered}
\left(x^{\left[k_{1}\right]}, \ldots, x^{\left[k_{m}\right]}\right)_{\circ}=x^{\left[k_{1}+\ldots+k_{m}+1\right]}, \text { for all } k_{1}, \ldots, k_{m} \in \mathbb{N} \\
\left(x^{[k]}\right)^{[s]}=x^{[k s(m-1)+k+s]}, \forall k, s \in \mathbb{N}
\end{gathered}
$$

We remark that

$$
\left(x^{[1]}\right)^{[1]}=x^{[m+1]}=\left(\stackrel{\left(m^{2}\right)}{x}\right)_{\circ} ;\left(x^{[m+1]}\right)^{[1]}=\left(\stackrel{\left(m^{3}\right)}{x}\right)_{\circ}=x^{\left[m^{2}+m+1\right]}
$$

and

$$
\left.\left(\stackrel{m^{k}}{x}\right)\right)_{\circ}=x^{\left[m^{k-1}+m^{k-2}+\ldots+m+1\right]}, \forall k \in \mathbb{N}^{*} .
$$

An $m$-semigroup $(S,()$ o) will be called:

- commutative, iff $\left(x_{1}^{m}\right)_{\circ}=\left(x_{\sigma(1)}^{\sigma(m)}\right)$ 。 for all $x_{1} \ldots, x_{m} \in S$ and for each permutation $\sigma$ of $1,2, \ldots, m$;

- semicommutative, iff $\left(x_{1}^{m}\right)_{\circ}=\left(x_{m} x_{2}^{m-1} x_{1}\right)_{\circ}$ for all $x_{1}, x_{2}, \ldots, x_{m} \in S$;

- entropic, iff $\left(\left(x_{11}^{1 m}\right)_{\circ}\left(x_{21}^{2 m}\right)_{\circ} \ldots\left(x_{m 1}^{m m}\right)_{\circ}\right)_{\circ}=\left(\left(x_{11}^{m 1}\right)_{\circ},\left(x_{12}^{m 2}\right)_{\circ}, \ldots,\left(x_{1 m}^{m m}\right)_{\circ}\right) \circ$ for any $m$ by $m$ matrix $\left(x_{i j}\right)_{i, j=1, m}$ of elements from $S$;

- normal (or exponential), iff $\left(x_{1}^{m}\right)_{\circ}^{[n]}=\left(x_{1}^{[n]}, \ldots, x_{m}^{[n]}\right)$ 。 for all $x_{1}, \ldots, x_{m} \in S$ and for all $n \in \mathbb{N}$.

We remark that commutativity implies semicommutativity, semicommutativity implies entropy and entropy implies the normality of $m$-semigroup $S$.

An $m$-semigroup $\left(S,()_{\circ}\right)$ is called an $m$-group if for all $a_{0}, a_{1}, \ldots, a_{m} \in S$ and fixed $i \in\{1, \ldots, m\}$ there exists an unique element $x \in S$ such that $\left(a_{1}^{i-1}, x, a_{i+1}^{m}\right)$ 。 $=a_{0}$. In some $m$-groups there is an element $e \in A$ (called an identity or a neutral element) such that $(\stackrel{(i-1)}{e}, x, \stackrel{(m-i)}{e}) \circ=x$ holds for all $x \in S$ and for all $i=1, \ldots, m$. It is interesting that there are $m$-groups with two or more neutral elements or which do not contain such elements [7,16].

We mention that if $(S, *)$ is a semigroup (group) and we define the $m$ - ary operation ()。: $S^{m} \rightarrow S,\left(x_{i}^{m}\right)_{\circ}=x_{1} * x_{2} \cdots * x_{m}$, then $\left(S,()_{\circ}\right)$ is an $m$-semigroup ( $m$-group), so called the $m$-semigroup ( $m$-group) derived from the binary semigroup (group) $(S, *)$. In addition, there are $m$-semigroups that are not derived from a binary semigroup.

Definition 1. A normed $m$-semigroup is a triplet $(S,() \circ,\|\|)$ such that $(S,() \circ)$ is an $m$-semigroup and \|\|$: S \rightarrow[0, \infty),\left\|\left(x_{1}^{m}\right)_{\circ}\right\| \leq\left\|x_{1}\right\|+\ldots+\left\|x_{m}\right\|$ for all $x_{1}, \ldots, x_{m} \in S$. 
Definition 2. Let $(X, *,\|\|)$ be a Banach space over the field of real numbers or complex numbers. If we define an m-ary operation ()$_{\circ}: X^{m} \rightarrow X ;\left(x_{1}^{m}\right)_{\circ}=$ $x_{1} * x_{2} \cdots * x_{m}$ then $\left(X,()_{\circ}\right)$ is an commutative $m-$ group with neutral element. We say that $\left(X,()_{\circ},\|\|\right)$ is an $m$-ary Banach space derived from $(X, *,\|\|)$.

Extending the notion of ternary Banach algebra given in [5],[23] we have

Definition 3. An m-ary Banach algebra is a normed Banach space $(X,\|\|)$ equipped with an m-ary operation ()$_{\circ}: X^{m} \rightarrow X$ which is associative and satisfies $\left\|\left(x_{1}^{m}\right)_{\circ}\right\| \leq\left\|x_{1}\right\| \ldots\left\|x_{m}\right\|$ for all $x_{1}, \ldots, x_{m} \in S$. If $\left\|\left(x_{1}^{m}\right)_{\circ}\right\|=\left\|x_{1}\right\| \ldots\left\|x_{m}\right\|$ then we say that the norm is multiplicative.

\section{THE GENERALIZED HyERS-Ulam STABILITy}

P. Găvruţă [12] obtained a generalization of Th. M. Rassias's theorem by replacing the Cauchy differences by a control mapping $\varphi$ satisfying a condition of convergence. In the same manner, using the Hyers "direct method" we can prove the following theorem, which generalizes Theorem 2.1 of Amyari and Moslehian [1] for ternary semigroups.

Theorem 1. Let $\left(S,()_{\circ}\right)$ be an $m$-semigroup, $X$ a Banach space and $\varphi: S^{m} \rightarrow[0, \infty)$ be a mapping such that

$$
\left.\tilde{\varphi}\left(x_{1}, \ldots, x_{m}\right):=\frac{1}{m} \sum_{n=0}^{\infty} m^{-n} \varphi\left(\left(\stackrel{(m}{x}_{1}^{n}\right)\right)_{\circ}, \ldots,\left(\stackrel{m^{n}}{x_{m}}\right) \text { 。 }\right)<\infty
$$

Suppose that the map $f: S \rightarrow X$ satisfies the condition

$$
\left\|f\left(\left(x_{1}, \ldots, x_{m}\right)_{\circ}\right)-\sum_{i=1}^{m} f\left(x_{i}\right)\right\| \leq \varphi\left(x_{1}, \ldots, x_{m}\right) .
$$

Then there exists an unique mapping $T: S \rightarrow X$ such that

$$
\|f(x)-T(x)\| \leq \tilde{\varphi}(x, x, \ldots, x)
$$

and

$$
T\left(x^{[1]}\right)=m T(x), \text { for all } x \in S .
$$

Moreover, if $S$ is a normal $m$-semigroup then $T$ is a homomorphism of $S$ on $\left(X, \sum\right)$, the $m$-semigroup derived from $(X,+)$.

Proof. For $x_{1}=\ldots=x_{m}=x$ in (3.2) we get

$$
\left\|f\left(x^{[1]}\right)-m f(x)\right\| \leq \varphi(x, \ldots, x)
$$

or

$$
\left\|\frac{1}{m} f\left(x^{[1]}\right)-f(x)\right\| \leq \frac{1}{m} \varphi(x, \ldots, x) .
$$


Because

$$
\left.\left(\begin{array}{c}
m^{k+1} \\
x
\end{array}\right) \text { 。 }=x^{\left[m^{k}+m^{k-1}+\ldots+m+1\right]}=\left(x^{\left[m^{k-1}+m^{k-2}+\ldots+1\right]}\right)^{[1]}=\left(\left(\stackrel{(m}{k}^{k}\right)\right)_{\circ}\right)^{[1]}
$$

we also have

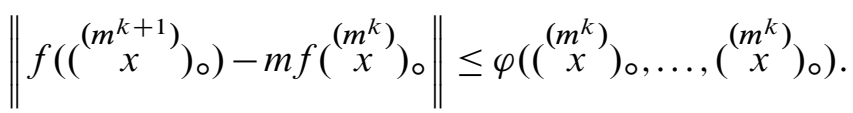

Then, recursively, we obtain

$$
\begin{aligned}
& \left\|m^{-n} f\left(\left(\begin{array}{c}
\left.m^{n}\right) \\
x
\end{array}\right) \circ\right)-f(x)\right\|=\left\|\sum_{k=0}^{n-1}\left(m^{-(k+1)} f\left(\left(\begin{array}{c}
m^{k+1} \\
x
\end{array}\right) \circ\right)-m^{-k} f\left(\left(\stackrel{(m}{k}^{k}\right) \circ\right)\right)\right\| \\
& =\| \sum_{k=0}^{n-1} m^{-k}\left(\frac { 1 } { m } f \left(\left(\left(^{k+1} x\right) \circ\right)-f\left(\left(\left(^{k} x\right) \circ\right)\right) \|\right.\right. \\
& \leq \sum_{k=0}^{n-1} m^{-k} \| \frac{1}{m} f\left(\left(\left(^{k+1} x\right) \text { 。 }\right)-f\left(\left(\left(^{k} x\right) \text { 。 }\right) \|\right.\right. \\
& \leq \frac{1}{m} \sum_{k=0}^{n-1} m^{-k} \varphi\left(x^{\left[\sum_{i=0}^{k-1} m^{i}\right]}, \ldots, x^{\left[\sum_{i=0}^{k-1} m^{i}\right]}\right) \text {. }
\end{aligned}
$$

Hence

$$
\left\|m^{-n} f\left(x^{\left[\sum_{i=0}^{n-1} m^{i}\right]}\right)-f(x)\right\| \leq \frac{1}{m} \sum_{k=0}^{n-1} m^{-k} \varphi\left(x^{\left[\sum_{i=0}^{k-1} m^{i}\right]}, \ldots, x^{\left[\sum_{i=0}^{k-1} m^{i}\right]}\right) .
$$

Similarly, for all $x \in S, n, r \in \mathbb{N} ; r<n$ we can show that

$$
\begin{aligned}
& \left\|m^{-n} f\left(x^{\left[\sum_{i=0}^{n-1} m^{i}\right]}\right)-m^{-r} f\left(x^{\left[\sum_{i=0}^{r-1} m^{i}\right]}\right)\right\| \\
\leq & \frac{1}{m} \sum_{k=r}^{n-1} m^{-k} \varphi\left(x^{\left[\sum_{i=0}^{k-1} m^{i}\right]}, \ldots, x^{\left[\sum_{i=0}^{k-1} m^{i}\right]}\right) .
\end{aligned}
$$

From this inequality and condition (3.1) we conclude that the sequence

$$
a_{n}(x)=m^{-n} f\left(x^{\left[\sum_{i=0}^{n-1} m^{i}\right]}\right)
$$

(a so called generalized Hyers-Ulam sequence) is a Cauchy sequence in $X$. In view of above conclusion, corroborated with the completeness of $X$, it follows that $\left\{a_{n}(x)\right\}_{n \in \mathbb{N}}$ is convergent. Let $T: S \rightarrow X$ be their limit

$$
T(x)=\lim _{n \rightarrow \infty} m^{-n} f\left(x^{\left[\sum_{i=0}^{n-1} m^{i}\right]}\right) .
$$


Because $\left(x^{[1]}\right)^{\left[\sum_{i=0}^{n-1} m^{i}\right]}=x^{\left[\sum_{i=0}^{n} m^{i}\right]}$, for all $x \in S$, we obtain

$$
\begin{aligned}
& T\left(x^{[1]}\right)=\lim _{n \rightarrow \infty} m^{-n} f\left(\left(x^{[1]}\right)^{\left[\sum_{i=0}^{n-1} m^{i}\right]}\right)= \\
= & m \lim _{n \rightarrow \infty} m^{-(n+1)} \underset{n \rightarrow \infty}{f}\left(x^{\left[\sum_{i=0}^{n} m^{i}\right]}\right)=m T(x) .
\end{aligned}
$$

By (3.1) and (3.6) and by letting $n \rightarrow \infty$ in inequality (3.5), we have

$$
\|T(x)-f(x)\| \leq \tilde{\varphi}(x, \ldots, x)
$$

for all $x \in S$.

We intend now to prove that $T$ is uniquely defined. Let $T^{\prime}: S \rightarrow X$ be another mapping with these properties. Then by (3.4) we conclude

$$
\begin{aligned}
\left\|T(x)-T^{\prime}(x)\right\| & =m^{-n}\left\|m^{n} T(x)-m^{n} T^{\prime}(x)\right\| \\
& =m^{-n}\left\|T\left(x^{\left[\sum_{i=0}^{n-1} m^{i}\right]}\right)-T^{\prime}\left(x^{\left[\sum_{i=0}^{n-1} m^{i}\right]}\right)\right\| \\
& \leq m^{-n}\left\|T\left(x^{\left[\sum_{i=0}^{n-1} m^{i}\right]}\right)-f\left(x^{\left[\sum_{i=0}^{n-1} m^{i}\right]}\right)\right\|+ \\
& +m^{-n}\left\|f\left(x^{\left[\sum_{i=0}^{n-1} m^{i}\right]}\right)-T^{\prime}\left(x^{\left[\sum_{i=0}^{n-1} m^{i}\right]}\right)\right\|
\end{aligned}
$$

and by (3.3) we have

$$
\left\|T(x)-T^{\prime}(x)\right\| \leq 2 m^{-n} \tilde{\varphi}\left(x^{\left[\sum_{i=0}^{n-1} m^{i}\right]}, \ldots, x^{\left[\sum_{i=0}^{n-1} m^{i}\right]}\right) .
$$

Taking the limit in this inequality as $n \rightarrow \infty$, we obtain $T(x)=T^{\prime}(x)$ for all $x \in S$ and so $T$ is unique.

In the sequel, we suppose that $\left(S,()_{\circ}\right)$ is a normal $m$-semigroup, i.e.

$$
\left(x_{1}, \ldots, x_{m}\right)_{\circ}^{[k]}=\left(x_{1}^{[k]}, \ldots, x_{m}^{[k]}\right)_{\circ}
$$

for all $x_{1}, \ldots, x_{m} \in S$ and all $k \in \mathbb{N}$. Replacing $x_{j}$ by $x_{j}^{\left[\sum_{i=0}^{n-1} m^{i}\right]}, j=1, \ldots, m$ in inequality (3.2) we have

$$
\begin{gathered}
\left\|f\left(\left[x_{1}^{\left[\sum_{i=0}^{n-1} m^{i}\right]}, \ldots, x_{m}^{\left[\sum_{i=0}^{n-1} m^{i}\right]}\right]_{\circ}\right)-\sum_{j=1}^{m} f\left(x_{j}^{\left[\sum_{i=0}^{n-1} m^{i}\right]}\right)\right\| \\
\leq \varphi\left(x_{1}^{\left[\sum_{i=0}^{n-1} m^{i}\right]}, \ldots, x_{m}^{\left[\sum_{i=0}^{n-1} m^{i}\right]}\right) .
\end{gathered}
$$

Using the equality (3.7), if we divide both sides by $m^{n}$ we obtain

$$
\begin{gathered}
\left\|m^{-n} f\left(\left(x_{1}, \ldots, x_{m}\right)_{\circ}^{\left[\sum_{i=0}^{n-1} m^{i}\right]}\right)-\sum_{j=1}^{m} m^{-n} f\left(x_{j}^{\left[\sum_{i=0}^{n-1} m^{i}\right]}\right)\right\| \\
\leq m^{-n} \varphi\left(x_{1}^{\left[\sum_{i=0}^{n-1} m^{i}\right]}, \ldots, x_{m}^{\left[\sum_{i=0}^{n-1} m^{i}\right]}\right) .
\end{gathered}
$$

In the last inequality, by letting $n \rightarrow \infty$, and by (3.1) and (3.6), we get 


$$
T\left(\left(x_{1}, \ldots, x_{m}\right)_{\circ}\right)=\sum_{j=1}^{m} T\left(x_{j}\right)
$$

i.e. $T$ is an $m-$ semigroup homomorphism.

Therefore, we have proven that under suitable conditions on the function involved, "near" any solution of inequality (3.2) there exists a solution of equation $f\left(\left(x_{1}^{m}\right)_{\circ}\right)=$ $\sum_{j=1}^{m} f\left(x_{j}\right)$. The word "near" means that the distance of the solution of the equation from the solution of the inequality is explicitly evaluated through the function $\varphi$ : $S^{m} \rightarrow[0, \infty)$.

As a consequence, if $\varphi\left(x_{1}, \ldots, x_{m}\right)=$ constant, Theorem 1 gives a generalization of the well known Hyers result [13].

Corollary 1. Let $\left(S,()_{\circ}\right)$ be an $m$-semigroup, $m \geq 2, X$ be a Banach space and $\varepsilon>0$. Suppose that $f: S \rightarrow X$ is a mapping satisfying

for all $x_{1}, \ldots, x_{m} \in S$.

$$
\left\|f\left(\left(x_{1}, \ldots, x_{m}\right)_{\circ}\right)-\sum_{j=1}^{m} f\left(x_{j}\right)\right\|<\varepsilon
$$

Then there exists an unique mapping $T: S \rightarrow X$ such that

and

$$
\|f(x)-T(x)\|<\frac{\varepsilon}{m-1}
$$

$$
T\left(x^{[1]}\right)=m T(x), \forall x \in S .
$$

Moreover, if $S$ is a normal $m$-semigroup, then $T$ is an m-semigroup homomorphism.

Proof. Because $m \geq 2$, the series $\sum_{n=0}^{\infty} \frac{\varepsilon}{m^{n+1}}$ is convergent with sum $\frac{\varepsilon}{m-1}$. Applying Theorem 1 there exists an unique mapping $T: S \rightarrow X$ such that

$$
T(x)=\lim _{n \rightarrow \infty} m^{-n} f\left(x^{\left[m^{n-1}+\ldots+m+1\right]}\right)
$$

with

$$
T\left(x^{[1]}\right)=m T(x) ; \forall x \in S
$$

and

$$
\|f(x)-T(x)\|<\frac{\varepsilon}{m-1} .
$$

For various forms of the function $\varphi\left(x_{1}, \ldots, x_{m}\right)$ we obtain other generalizations of some results on stability published in the last years.

First, we suppose that $f: S \rightarrow X$ is a mapping satisfying a condition weaker than Hyers's condition on approximate additive mappings in terms of a product of powers of norms. 
Corollary 2. Let $\left(S,()_{\circ},\|\|_{1}\right)$ be a normed $m$-semigroup, $m \in \mathbb{N}, m \geq 2,\left(X,\|\|_{2}\right)$ be a Banach space and $\varepsilon>0$. Suppose that $k_{1}, \ldots, k_{m} \in \mathbb{R} ; 0 \leq p=k_{1}+\ldots+k_{m}<1$ and $f: S \rightarrow X$ is a mapping satisfying the condition

$$
\begin{gathered}
\left\|f\left(\left(x_{1}, \ldots, x_{m}\right)_{\circ}\right)-\sum_{j=1}^{m} f\left(x_{j}\right)\right\|_{2}<\varepsilon\left\|x_{1}\right\|_{1}^{k_{1}}\left\|x_{2}\right\|_{1}^{k_{2}} \ldots\left\|x_{m}\right\|_{1}^{k_{m}}, \\
\forall x_{1}, \ldots, x_{m} \in S .
\end{gathered}
$$

Then there is an unique mapping $T: S \rightarrow X$ such that

and

$$
\|f(x)-T(x)\|_{2}<\frac{\varepsilon\|x\|_{1}^{p}}{m-m^{p}}
$$

$$
T\left(x^{[1]}\right)=m T(x), \forall x \in S .
$$

Moreover, if $S$ is a normal $m$-semigroup, then $T$ is an m-ary homomorphism.

Proof. Because $\left(S,()_{\circ},\|\|_{1}\right)$ is a normed $m$-semigroup, we have for all $x \in S$,

$$
\left\|x^{[1]}\right\|_{1}=\left\|(\stackrel{m}{x}){ }_{0}\right\|_{1} \leq m\|x\|_{1} .
$$

By induction, we have $\left\|x^{[k]}\right\|_{1} \leq((m-1) k+1)\|x\|_{1}$ for all $k \in \mathbb{N}$.

Hence

$$
\left\|x^{\left[m^{n-1}+\ldots+m+1\right]}\right\|_{1} \leq m^{n}\|x\|_{1}
$$

for all $n \in \mathbb{N}$ and all $x \in S$.

Let $\varphi: S^{m} \rightarrow[0, \infty)$ be defined by $\varphi\left(x_{1}, \ldots, x_{m}\right)=\varepsilon\left\|x_{1}\right\|_{1}^{k_{1}} \ldots\left\|x_{m}\right\|_{1}^{k_{m}}$ where $0 \leq p=k_{1}+\ldots+k_{m}<1$.

Because

$$
\begin{aligned}
& \left.\varphi\left(\left(\stackrel{(m}{x}_{1}^{n}\right)\right)_{0}, \ldots,\left(\stackrel{\left(m^{n}\right)}{x_{m}}\right) \circ\right)=\varepsilon\left\|\left({\left.\stackrel{(m}{x_{1}}\right)}^{n}\right) \circ\right\|_{1}^{k_{1}} \ldots\left\|\left(\stackrel{\left(m^{n}\right)}{x_{m}}\right)\right\|_{1} \|_{1}^{k_{m}} \\
& \leq \varepsilon\left(m^{n}\left\|x_{1}\right\|_{1}\right)^{k_{1}} \ldots\left(m^{n}\left\|x_{m}\right\|_{1}\right)^{k_{m}} \\
& =\varepsilon\left(m^{n}\right)^{k_{1}+\ldots+k_{m}}\left\|x_{1}\right\|_{1}^{k_{1}} \ldots\left\|x_{m}\right\|_{1}^{k_{m}} \\
& =\varepsilon m^{n p}\left\|x_{1}\right\|_{1}^{k_{1}} \ldots\left\|x_{m}\right\|_{1}^{k_{m}} \text {, }
\end{aligned}
$$

the series

$$
\frac{1}{m} \sum_{n=0}^{\infty} m^{-n} \varphi\left(\left(\stackrel{m}{x}_{1}^{n}\right) \text { ○ } \ldots\left(\stackrel{\left(m^{n}\right)}{x_{m}}\right) \circ\right) \leq \frac{1}{m} \sum_{n=0}^{\infty} \varepsilon\left(m^{p-1}\right)^{n}\left\|x_{1}\right\|_{1}^{k_{1}} \ldots\left\|x_{m}\right\|_{1}^{k_{m}}<\infty
$$

is convergent with the sum

and

$$
\widetilde{\varphi}\left(x_{1}, \ldots, x_{m}\right) \leq \frac{\varepsilon}{m-m^{p}}\left\|x_{1}\right\|_{1}^{k_{1}} \ldots\left\|x_{m}\right\|_{1}^{k_{m}}
$$




$$
\widetilde{\varphi}(x, \ldots, x) \leq \frac{\varepsilon\|x\|_{1}^{p}}{m-m^{p}} .
$$

According to Theorem 1 there is an unique mapping $T: S \rightarrow X$ such that

$$
\|f(x)-T(x)\|_{2} \leq \frac{\varepsilon\|x\|_{1}^{p}}{m-m^{p}}
$$

and

$$
T\left(x^{[1]}\right)=m T(x), \text { for all } x \in S .
$$

If $S$ is a normal normed $m$-semigroup, then $T$ is an $m$-ary homomorphism.

We remark that, in the special case where $m=2$, Corollary 2 is Theorem 1.1 from J. M. Rassias [18].

Corollary 3. Let $\left(S,() \circ,\|\|_{1}\right)$ be a normed $m$-semigroup $m \in \mathbb{N}, m \geq 2,\left(X,\|\|_{2}\right)$ be an $m$-ary Banach space and $\varepsilon>0$. Suppose that $f: S \rightarrow X$ is a mapping satisfying the inequality

$$
\left\|f\left(x_{1}, x_{2}, \ldots, x_{m}\right)_{\circ}-\sum_{j=1}^{m} f\left(x_{j}\right)\right\|_{2}<\varepsilon\left(\left\|x_{1}\right\|_{1}^{p}+\ldots+\left\|x_{m}\right\|_{1}^{p}\right)
$$

for $x_{1}, \ldots, x_{m} \in S$, where $0 \leq p<1$.

Then there is an unique mapping $T: S \rightarrow X$ such that

$$
\|f(x)-T(x)\|_{2} \leq \frac{\varepsilon m\|x\|_{1}^{p}}{m-m^{p}}
$$

and

$$
T\left(x^{[1]}\right)=m T(x), \forall x \in S .
$$

Moreover, if $S$ is a normal $m$-semigroup, then $T$ is an $m$-ary homomorphism.

Proof. We apply Theorem 1 to the mapping $\varphi: S^{m} \rightarrow X$,

$$
\varphi\left(x_{1}, \ldots, x_{m}\right)=\varepsilon\left(\left\|x_{1}\right\|_{1}^{p}+\ldots+\left\|x_{m}\right\|_{1}^{p}\right) .
$$

Because for $0 \leq p<1$, the series $\sum_{n=0}^{\infty} m^{n(p-1)}$ converges to $\frac{m}{m-m^{p}}$ and so we obtain the result above.

In particular, the case $m=2$ gives the results of T. Aoki [3] and Th. M. Rassias [19]. 


\section{Superstability}

Let $(S,()$ 。) be an $m$-semigroup, $X$ a Banach space and $f: S \rightarrow X$ be a mapping. Investigations concerning stability properties of the functional equation

$$
f\left(\left(x_{1}^{m}\right)_{\circ}\right)=\sum_{j=1}^{m} f\left(x_{j}\right)
$$

proved that any "approximate" solution of this equation can be approximated by an exact solution, i.e., by an $m$-ary homomorphism if we regard $\left(X, \sum\right)$ as an $m$-semigroup derived from $(X,+)$. The equation $f\left(\left(x_{1}^{m}\right)_{\circ}\right)=\prod_{j=1}^{m} f\left(x_{j}\right)$ possesses a more surprising stability property, namely, any unbounded approximate solution must be an $m$-ary homomorphism. Such a phenomenon is called superstability (see L. Szekelyhidi [21], for $m=2$ and M. Amyari and M.S. Moslehian [1] for $m=3$ ). More exactly, suppose that we are given a functional equation $E(f)=0$, such that the notion of boundedness of $f$ and $E(f)$ makes sense and furthermore, we assume that $E(f)$ is bounded whenever $f$ is bounded. The functional equation $E(f)=0$ is stable if any function $g$ approximately satisfying this equation is near to a true solution of this equation. This functional equation is superstable if the boundedness of $E(f)$ implies that either $f$ is bounded or $E(f)=0$. Therefore the notion of superstability is "stronger" than the notion of stability.

In this section we will study the superstability of $m$-ary homomorphism, generalizing the results of Amyari and Moslehian. [1].

Theorem 2. Let $\left(S,()_{\circ}\right)$ be an $m$-semigroup, A a normed algebra whose norm is multiplicative and $\varepsilon \geq 0$. If $f: S \rightarrow A$ satisfies the inequality

$$
\left\|f\left(\left(x_{1}, \ldots, x_{m}\right)_{\circ}\right)-\prod_{j=1}^{m} f\left(x_{j}\right)\right\| \leq \varepsilon
$$

for all $x_{1}, \ldots, x_{m} \in S$, then there is $\delta_{\varepsilon}>1$ such that either

$$
\|f(x)\| \leq \delta_{\varepsilon}, \text { for all } x \in S,
$$

or

$$
f\left(\left(x_{1}, \ldots, x_{m}\right)_{\circ}\right)=\prod_{j=1}^{m} f\left(x_{j}\right), \forall x_{1}, \ldots, x_{m} \in S .
$$

Proof. By inequality (4.1) we have $\left\|f\left(x^{[1]}\right)-(f(x))^{m}\right\| \leq \varepsilon$.

We remark that the equation $\delta^{m}-\delta=\varepsilon$ has a unique solution $\delta_{\varepsilon}>1$. Suppose that there exists $a \in S$ such that $\|f(a)\|>\delta_{\varepsilon}$ i.e. $\|f(a)\|=\delta_{\varepsilon}+p$ for some $p>0$. Then

$$
\begin{aligned}
\left\|f\left(a^{[1]}\right)\right\| & =\left\|(f(a))^{m}-\left((f(a))^{m}-f\left(a^{[1]}\right)\right)\right\| \\
& \geq\|f(a)\|^{m}-\left\|(f(a))^{m}-f\left(a^{[1]}\right)\right\|
\end{aligned}
$$




$$
\begin{aligned}
& \geq\left(\delta_{\varepsilon}+p\right)^{m}-\varepsilon \\
& =\delta_{\varepsilon}^{m}+m \delta_{\varepsilon}^{m-1} p+\ldots+p^{m}-\left(\delta_{\varepsilon}^{m}-\delta_{\varepsilon}\right) \\
& >\delta_{\varepsilon}+m p .
\end{aligned}
$$

Assume by induction that

$$
\left\|f\left(a^{\left[m^{n-1}+\ldots+m+1\right]}\right)\right\|>\delta_{\varepsilon}+m^{n} p .
$$

Then

$$
\begin{aligned}
\left\|f\left(a^{\left[m^{n}+\ldots+m+1\right]}\right)\right\| & =\left\|f\left(\left(a^{\left[m^{n-1}+\ldots+m+1\right]}\right)^{[1]}\right)\right\| \\
& \geq\left\|f\left(a^{\left[m^{n-1}+\ldots+m+1\right]}\right)\right\|^{m}- \\
& -\left\|\left(f\left(a^{\left[m^{n-1}+\ldots+m+1\right]}\right)\right)^{m}-f\left(\left(a^{\left[m^{n-1}+\ldots+m+1\right]}\right)^{[1]}\right)\right\| \\
& \geq\left(\delta_{\varepsilon}+m^{n} p\right)^{m}-\varepsilon>\delta_{\varepsilon}+m^{n+1} p .
\end{aligned}
$$

Therefore

$$
\left\|f\left(a^{\left[m^{n-1}+\ldots+m+1\right]}\right)\right\|>\delta_{\varepsilon}+m^{n} p
$$

holds for all positive integers $n$.

Because for every $x_{1}, x_{2}, \ldots, x_{2 m-1} \in S$ according to the inequality (4.1) we have

$$
\left.\| f\left(\left(x_{1}^{m}\right)_{\circ} x_{m+1}^{2 m-1}\right)_{\circ}\right)-f\left(\left(x_{1}^{m}\right)_{\circ}\right) f\left(x_{m+1}\right) \ldots f\left(x_{2 m-1}\right) \| \leq \varepsilon
$$

and

$$
\left\|f\left(\left(x_{1}^{m-1}\left(x_{m}^{2 m-1}\right)_{\circ}\right) \circ\right)-f\left(x_{1}\right) \ldots f\left(x_{m-1}\right) f\left(\left(x_{m}^{2 m-1}\right)_{\circ}\right) \circ\right\| \leq \varepsilon .
$$

By associativity of $m$-ary operation (), we obtain

$$
\| f\left(\left(x_{1}^{m}\right) \circ f\left(x_{m+1}\right) \ldots f\left(x_{2 m-1}\right)-f\left(x_{1}\right) \ldots f\left(x_{m-1}\right) \circ f\left(\left(x_{m}^{2 m-1}\right) \circ\right) \| \leq 2 \varepsilon .\right.
$$

Because the norm \|\| is multiplicative, we have

$$
\begin{aligned}
&\left\|f\left(\left(x_{1}^{m}\right)_{\circ}\right) f\left(x_{m+1}\right) \ldots f\left(x_{2 m-1}\right)-\prod_{j=1}^{2 m-1} f\left(x_{j}\right)\right\| \\
& \leq\left\|f\left(\left(x_{1}^{m}\right)_{\circ}\right) f\left(x_{m+1}\right) \ldots f\left(x_{2 m-1}\right)-f\left(x_{1}\right) \ldots f\left(x_{m-1}\right) f\left(\left(x_{m}^{2 m-1}\right)_{\circ}\right)\right\|+ \\
&+\left\|f\left(x_{1}\right) \ldots f\left(x_{m-1}\right)\left(f\left(\left(x_{m}^{2 m-1}\right)_{\circ}\right)-f\left(x_{m}\right) \ldots f\left(x_{2 m-1}\right)\right)\right\| \\
& \leq 2 \varepsilon+\left\|f\left(x_{1}\right)\right\| \ldots\left\|f\left(x_{m-1}\right)\right\| \varepsilon . \\
& \text { In particular, for } x_{m+1}=x_{m+2}=\ldots=x_{2 m-1}=a^{\left[m^{n-1}+\ldots+m+1\right]} \text { we obtain }
\end{aligned}
$$

$$
\begin{gathered}
\left\|f\left(\left(x_{1}^{m}\right)_{\circ}\right)-\prod_{j=1}^{m} f\left(x_{j}\right)\right\| \cdot\left\|f\left(a^{\left[m^{n-1}+\ldots m+1\right]}\right)\right\|^{m-1} \\
\leq 2 \varepsilon+\left\|f\left(x_{1}\right)\right\| \ldots\left\|f\left(x_{m-1}\right)\right\| \varepsilon .
\end{gathered}
$$


By (4.4)

$$
\left\|f\left(\left(x_{1}^{m}\right)_{\circ}\right)-f\left(x_{1}\right) \ldots f\left(x_{m}\right)\right\| \leq \frac{2 \varepsilon+\left\|f\left(x_{1}\right)\right\| \ldots\left\|f\left(x_{m-1}\right)\right\| \varepsilon}{\left(\delta_{\varepsilon}+m^{n} p\right)^{m-1}} .
$$

Letting $n \rightarrow \infty$ then

$$
f\left(x_{1}^{m}\right)_{\circ}=f\left(x_{1}\right) \ldots f\left(x_{m}\right)
$$

for all $x_{1}, \ldots, x_{m} \in S$.

The Baker's example [4] extended to $m$-ary product shows that the crucial step in the proof above is the fact that the norm is multiplicative.

Given $\varepsilon>0$ there is an unique solution $\delta_{\varepsilon}$ greater than 1 of the equation $\left|\delta^{m}-\delta\right|=$ $\varepsilon$. If $(S=\mathbb{R},() \circ)$ is the $m$-semigroup with $\left(x_{1}^{m}\right)_{\circ}=x_{1}+\ldots+x_{m}, M_{2}(\mathbb{R})$ is the algebra of $2 \times 2$ matrices with real elements and $f: \mathbb{R} \rightarrow M_{2}(\mathbb{R}), f(x)=\left(\begin{array}{cc}e^{x} & 0 \\ 0 & \delta_{\varepsilon}\end{array}\right)$ then for the usual matrix norm we have

$$
\begin{gathered}
\left\|f\left(x_{1}+\ldots+x_{m}\right)-f\left(x_{1}\right) \ldots f\left(x_{m}\right)\right\|= \\
\quad=\left\|\left(\begin{array}{cc}
0 & 0 \\
0 & \delta-\delta^{m}
\end{array}\right)\right\|=\left|\delta-\delta^{m}\right|=\varepsilon,
\end{gathered}
$$

for all $x_{1}, \ldots, x_{n} \in \mathbb{R}$. Hence $f\left(x_{1}+\ldots+x_{m}\right) \neq f\left(x_{1}\right) \ldots f\left(x_{m}\right)$ while $f$ is unbounded.

Szekelyhidi [21] proved that the invariance of the vector space where the functions are defined is enough to ensure superstability. The notions and results of Theorem 2 can be generalized in the following:

Definition 4. Let $\left(S,()_{\circ}\right)$ be an $m$-semigroup and let $V$ be the linear space of complex $\mathbb{C}$-valued function on $S$. The space $V$ is called right (left) invariant if $\varphi: S \rightarrow \mathbb{C}$ belongs to $V$ implies that the right (left) translation

$$
\begin{gathered}
\varphi_{y_{1}^{m-1}}: S \rightarrow \mathbb{C} ; \varphi_{y_{1}^{m-1}}(x)=\varphi\left(\left(x, y_{1}^{m-1}\right)_{\circ}\right) \\
\left(y_{1}^{m-1} \varphi: S \rightarrow \mathbb{C} ; y_{1}^{m-1} \varphi(x)=\varphi\left(\left(y_{1}^{m-1}, x\right)_{\circ}\right)\right)
\end{gathered}
$$

belongs to $V$ for every $y_{1}, y_{2}, \ldots, y_{m-1} \in S$.

Theorem 3. Let $\left(S,()_{\circ}\right)$ be an $m$-semigroup, $V$ a right invariant linear space of complex valued functions on $S$ and $\varphi, f: S \rightarrow \mathbb{C}$ be nonzero functions for which the function $\psi_{y_{1}^{m-1}}: S \rightarrow \mathbb{C}$;

$$
\psi_{y_{1}^{m-1}}(x)=\varphi\left(\left(x, y_{1}^{m-1}\right)_{\circ}\right)-\varphi(x) f\left(y_{1}\right) \cdot \ldots \cdot f\left(y_{m-1}\right),
$$

belongs to $V$ for every $y_{1}, \ldots, y_{m-1} \in S$. Then either $\varphi \in V$, or $f$ is an $m$-ary homomorphism of $m$-semigroup $(S,() \circ)$ into $(\mathbb{C}, \Pi)$-the derived $m$-semigroup from the semigroup $(\mathbb{C}, \cdot)$. 
Proof. Suppose that $f$ is not an $m$-ary homomorphism of $S$ into $\mathbb{C}$. Hence there are elements $x_{2}, x_{3}, \ldots, x_{m+1} \in S$ such that

$$
f\left(\left(x_{2}^{m+1}\right)_{\circ}\right) \neq f\left(x_{2}\right) f\left(x_{3}\right) \ldots f\left(x_{m+1}\right)
$$

and therefore there is

$$
\left[f\left(\left(x_{2}^{m+1}\right)_{\circ}\right)-f\left(x_{2}\right) \ldots f\left(x_{m+1}\right)\right]^{-1} \stackrel{\text { denoted }}{=} a^{-1} .
$$

Assume that $x_{i} \in S$ such that $b_{i}=f\left(x_{i}\right) \neq 0 ; i \in\{m+2, \ldots, 2 m-1\}$.

Then, by associativity law of $m$-ary operation we have

$$
\begin{aligned}
& \varphi\left(\left(\left(x_{1}^{m}\right)_{\circ} x_{m+1}^{2 m-1}\right)_{\circ}\right)-\varphi\left(\left(x_{1}^{m}\right)_{\circ}\right) f\left(x_{m+1}\right) \ldots f\left(x_{2 m-1}\right) \\
& =\left[\varphi\left(\left(x_{1}\left(x_{2}^{m+1}\right)_{\circ} x_{m+2}^{2 m-1}\right)_{\circ}\right)-\varphi\left(x_{1}\right) f\left(\left(x_{2}^{m+1}\right)_{\circ}\right) f\left(x_{m+2}\right) \ldots f\left(x_{2 m-1}\right)\right] \\
& -\left[\varphi\left(\left(x_{1}^{m}\right)_{\circ}\right)-\varphi\left(x_{1}\right) f\left(x_{2}\right) \ldots f\left(x_{m}\right)\right] f\left(x_{m+1}\right) \ldots f\left(x_{2 m-1}\right) \\
& +\varphi\left(x_{1}\right)\left[f\left(\left(x_{2}^{m+1}\right)_{\circ}\right)-f\left(x_{2}\right) \ldots f\left(x_{m+1}\right)\right] f\left(x_{m+2}\right) \ldots f\left(x_{2 m-1}\right)
\end{aligned}
$$

and hence

$$
\begin{aligned}
& \varphi\left(x_{1}\right)=\left\{\left[\varphi\left(\left(\left(x_{1}^{m}\right)_{\circ} x_{m+1}^{2 m-1}\right)_{\circ}\right)-\varphi\left(\left(x_{1}^{m}\right)_{\circ}\right) f\left(x_{m+1}\right) \ldots f\left(x_{2 m-1}\right)\right]\right. \\
& -\left[\varphi\left(\left(x_{1}\left(x_{2}^{m+1}\right)_{\circ} x_{m+2}^{2 m-1}\right)_{\circ}\right)-\varphi\left(x_{1}\right) f\left(\left(x_{2}^{m+1}\right)_{\circ}\right) f\left(x_{m+2}\right) \ldots f\left(x_{2 m-1}\right)\right] \\
& \left.+\left[\varphi\left(\left(x_{1}^{m}\right)_{\circ}\right)-\varphi\left(x_{1}\right) f\left(x_{2}\right) \ldots f\left(x_{m}\right)\right] f\left(x_{m+1}\right) \ldots f\left(x_{2 m-1}\right)\right\} \\
& \cdot\left[f\left(x_{2}^{m+1}\right)-f\left(x_{2}\right) \ldots f\left(x_{m+1}\right)\right]^{-1}\left[f\left(x_{m+2}\right)\right]^{-1} \ldots\left[f\left(x_{2 m-1}\right)\right]^{-1} .
\end{aligned}
$$

Therefore

$$
\begin{aligned}
& \varphi\left(x_{1}\right)=\left[\psi_{x_{m+1}^{2 m-1}}\left(\left(x_{1}^{m}\right)_{\circ}\right)-\psi_{\left(x_{2}^{m+1}\right)_{\circ} x_{m+2}^{2 m-1}}\left(x_{1}\right)\right. \\
& \left.+\psi_{x_{2}^{m}}\left(x_{1}\right) f\left(x_{m+1}\right) \ldots f\left(x_{2 m-1}\right)\right] a^{-1} b_{m+2}^{-1} b_{m+3}^{-1} \ldots b_{2 m-1}^{-1} .
\end{aligned}
$$

Since $V$ is right-invariant linear space we conclude that the right-hand side of above equality as a function of $x_{1}$ belongs to $V$, then hence so does $\varphi$.

As a consequence we obtain a natural generalization of Theorem 3.4 of Amyari and Moslehian [1]:

Corollary 4. Let $\left(S,()_{\circ}\right)$ be an $m$-semigroup and $\varphi, f: S \rightarrow \mathbb{C}$ be nonzero functions for which there exists a bounded function $\alpha: S^{m-1} \rightarrow[0, \infty)$ such that

$$
\left|\varphi\left(\left(x_{1}^{m}\right)_{\circ}\right)-\varphi\left(x_{1}\right) f\left(x_{2}\right) \ldots f\left(x_{m}\right)\right| \leq \alpha\left(x_{2}, x_{3}, \ldots, x_{m}\right)
$$


for all $x_{1}, x_{2}, \ldots, x_{m} \in S$

Then either $\varphi$ is bounded or $f$ is an $m$-ary homomorphism of $(S,() \circ)$ into $(\mathbb{C}, \Pi)$.

Proof. Let $V$ be the linear space of all bounded $\mathbb{C}$-valued function on $S$. The function $\psi_{x_{2}^{m}}: S \rightarrow \mathbb{C}$

$$
\psi_{x_{2}^{m}}\left(x_{1}\right)=\varphi\left(\left(x_{1}^{m}\right)_{\circ}\right)-\varphi\left(x_{1}\right) f\left(x_{2}\right) \ldots f\left(x_{m}\right)
$$

belongs to $V$ for each $x_{2}, x_{3}, \ldots, x_{m}$. Then we can apply Theorem 3 which implies that either $\varphi$ is bounded or $f$ is an $m$-ary homomorphism.

In the special case where $f=\varphi: S \rightarrow \mathbb{C}$ and mapping $\alpha=\varepsilon, \varepsilon>0$ we obtain a generalization of Corollary 3.5 from [1]:

Corollary 5. Let $\left(S,()_{\circ}\right)$ be an $m$-semigroup, $\varepsilon>0$ and $f: S \rightarrow \mathbb{C}$ be a nonzero function such that

$$
\left|f\left(\left(x_{1}^{m}\right)_{\circ}\right)-\prod_{j=1}^{m} f\left(x_{j}\right)\right| \leq \varepsilon
$$

for all $x_{1}, x_{2}, \ldots, x_{m} \in S$.

Then either $f$ is bounded or $f$ is an m-ary homomorphism.

\section{ACKNOWLEDGEMENT}

We are thankful to the anonymous referees for theirs valuable suggestions that led to an improvement in the presentation of the paper.

\section{REFERENCES}

[1] M. Amyari and M. Moslehian, "Approximate homomorphisms of ternary semigroups," Lett. Math. Phys., vol. 77, no. 1, pp. 1-9, 2006.

[2] I. S. An and C. Park, "Isomorphisms and derivations in $C^{*}$-ternary algebras," Korean J. Math, vol. 17 , no. 1, pp. 83-90, 2009.

[3] T. Aoki, "On the stability of the linear transformation in Banach spaces," J. Math. Soc. Japan, vol. 2, pp. 64-66, 1950.

[4] J. Baker, "The stability of the cosine equation," Proc. Amer. Math. Soc., vol. 74, pp. 242-246, 1979.

[5] M. Dehghanian and M. S. Modarres, "Ternary Weighted Function and Beurling Ternary Banach Algebra $l_{1}^{\omega}(S)$, , Abs. and Appl. Analysis, p. 9 pages, 2011.

[6] M. Dehghanian and M. S. Modarres, "Ternary $\gamma$-homomorphisms and ternary $\gamma$-derivations on ternary semigroups," J. of Ineq. and Apply., p. 10 pages, 2012.

[7] W. Dörnte, "Untersuchungen über eine verallgemeinerten gruppenbegrieff," Math. Z., vol. 29, pp. $1-19,1928$.

[8] W. A. Dudek, "On divisibility in n-semigroups," Demonstratio Math., vol. 13, pp. 355-367, 1980.

[9] W. A. Dudek and K. Glazek, "Around the Hosszú-Gluskin theorem for $n$-groups," Discrete Math., vol. 308, pp. 4861-4876, 2008. 
[10] Z. Gajda, "On stability of additive mappings,” Internat. J. Math. Sci., vol. 14, no. 3, pp. 431-434, 1991.

[11] J. W. Grzymala-Busse, "Automorphisms of polyadic automata," J. Assoc. Comput. Mach, vol. 16, pp. 208-219, 1969.

[12] P. Găvruţa, "A generalization of the Hyers-Ulam-Rassian stability of approximately additive mappings," J. Math. Anal. Appl., vol. 184, pp. 431-436, 1994.

[13] D. H. Hyers, "On the stability of the linear functional equation," Proc. Nat. Acad. Sci. USA, vol. 27, pp. 222-224, 1941.

[14] R. Kerner, "Ternary and non-associative algebraic structures and their applications in physics," Proc. Conf. ICGTMP "Group-23”, 2000.

[15] M. S. Moslehian and L. Szekelyhidi, "Stability of ternary homomorphisms via generalized Jensen equation," Result. Math., vol. 49, pp. 286-300, 2006.

[16] E. L. Post, "Polyadic groups," Trans. Amer. Math. Soc., vol. 48, pp. 208-350, 1940.

[17] J. M. Rassias, "On approximation of approximately linear mappings by linear mappings," J. Funct. Anal., vol. 46, pp. 126-130, 1982.

[18] J. M. Rassias, "Solution of a problem of Ulam," J. Approx. Theory Math., vol. 57, pp. 268-273, 1989.

[19] T. M. Rassias, "On the stability of the linear mapping in Banach space," Proc. Amer. Math, Soc., vol. 72, pp. 297-300, 1978.

[20] T. M. Rassias, “The problem of S.M. Ulam for approximately multiplicative mappings," J. Math. Anal. Appl., vol. 246, pp. 352-378, 2000.

[21] L. Szekelyhidi, "On a theorem of Baker, Lawrence and Zorzitto," Proc. Amer. Math. Soc., vol. 84, pp. 95-96, 1982.

[22] S. M. Ulam, "A collection of mathematical problems," Interscience Tracts in Pure and Applied Mathematics, no. 8, 1960.

[23] H. Zettl, "A characterization of ternary rings of operators," Adv. Math., vol. 48, pp. 117-143, 1983.

Authors' addresses

\section{Adina Pop}

Technical University of Cluj Napoca, North Univ Center of Baia Mare, Department of Mathematics and Computer Science, 76 Victoriei Street, 430122 Baia Mare, Romania

E-mail address: adina_p_2006@yahoo.com

\section{Maria S. Pop}

Technical University of Cluj Napoca, North Univ Center of Baia Mare,, Department of Mathematics and Computer Science, 76 Victoriei Street, 430122 Baia Mare, Romania

E-mail address: maria_sanziana@yahoo.com 